\title{
No prolongation of skin allograft survival by immunoproteasome inhibition in mice
}

\author{
Sarah Mundt ${ }^{\mathrm{a}, \mathrm{b}, *}$, Michael Basler ${ }^{\mathrm{a}, \mathrm{c}}$, Birgit Sawitzki ${ }^{\mathrm{d}}$, Marcus Groettrup ${ }^{\mathrm{a}, \mathrm{c}, * * *}$ \\ a Division of Immunology, Department of Biology, Universitaetsstrasse 10, University of Konstanz, 78457 Konstanz, Germany \\ b Konstanz Research School Chemical Biology, University of Konstanz, Konstanz, Germany \\ c Biotechnology Institute Thurgau at the University of Konstanz (BITg), Unterseestrasse 47, 8280 Kreuzlingen, Switzerland \\ d Institute of Medical Immunology, Charite University Medicine, Augustenburger Platz 1, 13353 Berlin, Germany
}

\section{A R T I C L E I N F O}

\section{Keywords:}

Immunoproteasome

Immunoproteasome inhibitor

LMP7

MHC

miHA mismatch

Mouse skin transplantation

\begin{abstract}
A B S T R A C T
The immunoproteasome, a distinct class of proteasomes, which is inducible under inflammatory conditions and constitutively expressed in monocytes and lymphocytes, is known to shape the antigenic repertoire presented on major histocompatibility complex (MHC) class I molecules. Moreover, inhibition of the immunoproteasome subunit LMP7 ameliorates clinical symptoms of autoimmune diseases in vivo and was shown to suppress the development of T helper cell (Th) 1 and Th17 cells and to promote regulatory T-cell (Treg) generation independently of its function in antigen processing. Since Th1 and Th17 cells are detrimental and Treg cells are critical for transplant acceptance, we investigated the influence of the LMP7-selective inhibitor ONX 0914 in a mixed lymphocyte reaction (MLR) in vitro as well as on allograft rejection in a MHC-disparate (C57BL/6 to $\mathrm{BALB} / \mathrm{c}$ ) and a multiple minor histocompatibility antigen (miHA)-disparate (B10.Br to $\mathrm{C} 3 \mathrm{H}$ ) model of skin transplantation in vivo. Although we observed reduced allo-specific IL-17 production of T cells in vitro, we found that selective inhibition of LMP7 had neither an influence on allograft survival in an MHC-mismatch model nor in a multiple minor mismatch skin transplantation model. We conclude that inhibition of the immunoproteasome is not effective in prolonging skin allograft survival in skin allotransplantation.
\end{abstract}

\section{Introduction}

The $26 \mathrm{~S}$ proteasome is a multicatalytic protease in the nucleus and cytoplasm of all eukaryotic cells responsible for the ATP dependent degradation of the bulk ( $8090 \%$ ) of cellular proteins with critical functions in multiple biological processes (Goldberg et al., 2002). In cells of hematopoietic origin, or in response to interferon (IFN) $\gamma$, the constitutively expressed catalytically active $\beta$ subunits are replaced by their inducible counterparts low molecular mass polypeptide (LMP)2 ( $\beta 1 i)$, multicatalytic endopeptidase complex subunit (MECL) 1 ( $\beta 2 i)$, and LMP7 ( $\beta 5 \mathrm{i}$ ) during neosynthesis, thereby building the so called immunoproteasome (Groettrup et al., 2001). The incorporation of the inducible subunits leads to minor structural changes within the pro teasome, to a marked change in the cleavage preference, and to an enhanced production of $\mathrm{T}$ cell epitopes (Groettrup et al., 2001; Basler et al., 2013; Groettrup et al., 2010; Huber et al., 2012). Im munoproteasomes do not only play an important role in generating major histocompatibility complex (MHC) class I ligands for T cell ac tivation in the periphery but also in shaping the naive $\mathrm{T}$ cell repertoire in the thymus and regulating immune responses (Basler et al., 2013; Groettrup et al., 2010).

$\mathrm{CD} 4^{+} \mathrm{T}$ cells have long been known to play a central role in med iating transplant rejection (Jiang et al., 2004). Acute allograft rejection is a $\mathrm{T}$ cell dependent phenomenon and may be triggered by different types of $\mathrm{T}$ helper cells. $\mathrm{T}$ helper (Th) 1 cell responses initiate allograft rejection by promoting proliferation of alloreactive $\mathrm{CD} 8^{+} \mathrm{T}$ cells or by inducing a delayed type hypersensitivity (DTH) reaction mediated by macrophages. Additionally, Th1 cells promote transplant rejection by activating B cells to produce allo specific antibodies or directly through Fas/Fas ligand (Fas L) induced cytotoxicity (Jiang et al., 2004). In re cent years, it turned out that not only Th1 but also Th17 cells mediate acute allograft rejection by recruiting neutrophils and monocytes into the graft which then contributes to transplant inflammation (Itoh et al., 2011; Gorbacheva et al., 2010; Miura et al., 2003). Importantly, it has

Abbreviations: LMP, low molecular mass polypeptide; MECL, multicatalytic endopeptidase complex; miHA, minor histocompatibility antigen; MHC, major histocompatibility complex; CsA, cyclosporine A; MLR, mixed lymphocyte reaction

* Corresponding author at: Institute of Experimental Immunology, University of Zurich, Winterthurerstrasse 190, 8057 Zurich, Switzerland.

** Corresponding author at: Department of Biology, Division of Immunology, University of Konstanz, P1101 Universitaetsstrasse 10, 78457 Konstanz, Germany.

E-mail addresses: mundt@immunology.uzh.ch (S. Mundt), marcus.groettrup@uni-konstanz.de (M. Groettrup). 
a

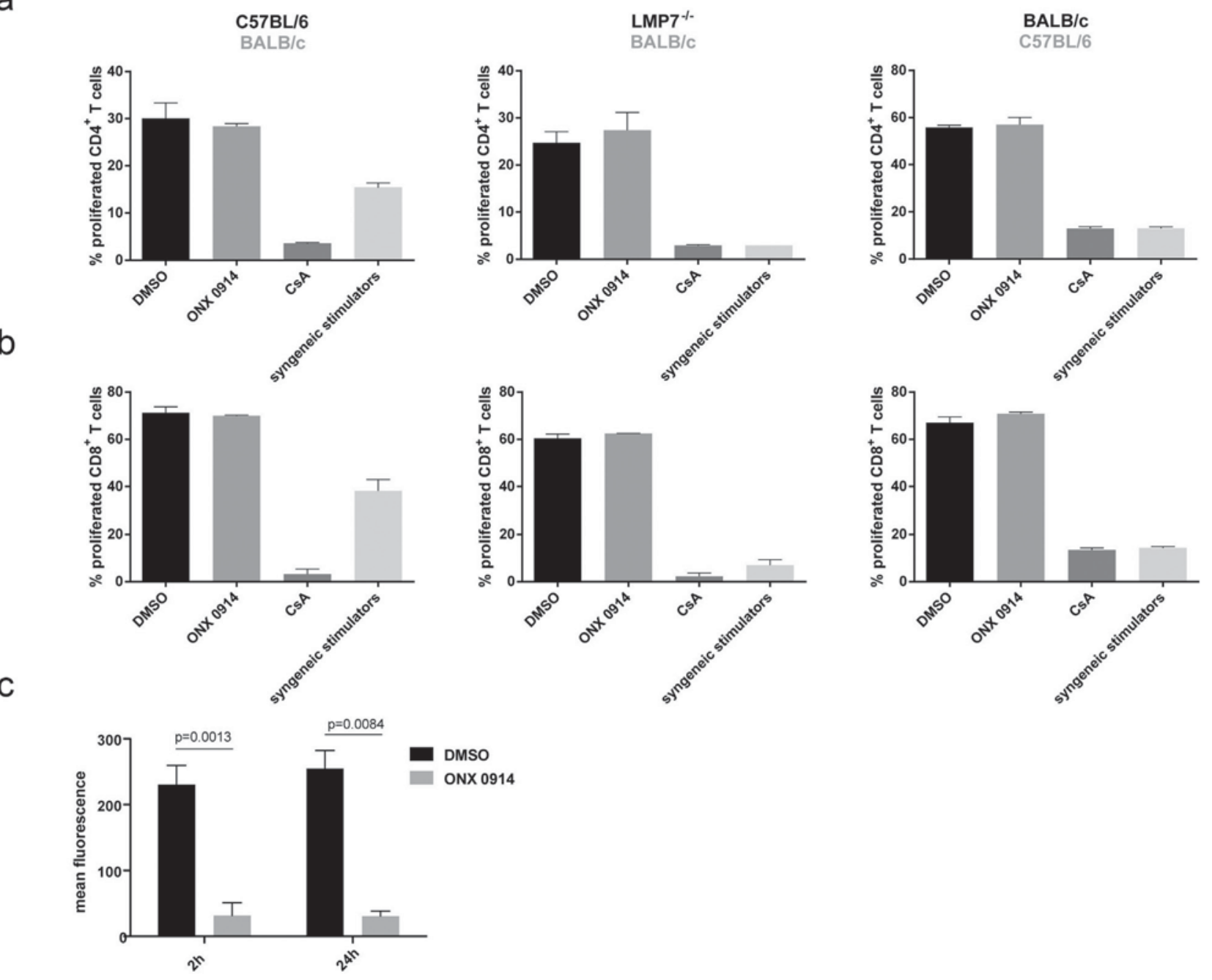

Fig. 1. Influence of LMP7 inhibition on allogeneic T-cell proliferation in a mixed lymphocyte reaction. CFSE labeled T cells magnetically isolated from bulk splenocytes of C57BL/6 (H$\left.2^{\mathrm{b}}\right)$, LMP7 $\left(\mathrm{H}-2^{\mathrm{b}}\right)$, and BALB/c $\left(\mathrm{H}-2^{\mathrm{d}}\right)$ mice (indicated in black above the panels) were treated with DMSO, ONX $0914(250 \mathrm{nM})$, or CsA (1 $\mu$ M) and cultured in the presence of allogeneic irradiated stimulator splenocytes (indicated in gray above the panels) for $96 \mathrm{~h}$. Syngeneic stimulator splenocytes were used as negative control. Graphs show proliferation of (a) $\mathrm{CD}^{+}$and (b) $\mathrm{CD}^{+} \mathrm{T}$ cells as measured by CFSE dilution. Data are presented as mean \pm SD from duplicates and represent one out of three independent experiments. Data were analyzed by student's $t$-test (two-tailed). (c) CFSE labeled T cells magnetically isolated from bulk splenocytes of C57BL/6 mice were treated with DMSO or ONX 0914 (250 nM) and cultured in the presence of allogeneic irradiated stimulators (BALB/c). $2 \mathrm{~h}$ and $24 \mathrm{~h}$ later, the proteasomal chymotrypsin-like activity in the cells was determined by the hydrolysis of the cell permeable substrate Meo-Suc-GLF-AMC. Depicted is the mean \pm SEM of the mean fluorescence of cells derived from three different mice measured in sextuplicates. The highest fluorescence value was set to $100 \%$. Data were analyzed by paired student's $t$-test (two-tailed).

been demonstrated that regulatory $\mathrm{T}$ cells (Tregs) induce and maintain tolerance to the allograft in experimental and clinical transplantation (Wood et al., 2012).

Bortezomib, a dipeptide boronate, is the first in class proteasome inhibitor approved for the treatment of multiple myeloma (Richardson et al., 2005). In addition, bortezomib was evaluated in clinical studies for solid tumors, including non small cell lung cancer (Li et al., 2010), and has demonstrated therapeutic efficacy in preventing chronic re jection of kidney grafts (Raghavan et al., 2010; Vogelbacher et al., 2010; Walsh et al., 2010). Most well characterized proteasome in hibitors mediate equivalent inhibition of both proteasome chymo trypsin like activities ( $\beta 5$ and LMP7) and have considerable toxicities that limit their clinical utility in chronic inflammatory diseases (Richardson et al., 2006). Immunoproteasomes are highly expressed in cells of hematopoietic origin implying that inhibitors specifically tar geting catalytically active immunosubunits might be a powerful tool for the treatment of inflammatory disorders while sparing other tissues. Their superiority in terms of drug resistance and toxicity may render immunoproteasome selective inhibitors as promising candidates for the treatment of allograft rejection. ONX 0914 (formerly designated PR
957) is an irreversible proteasome inhibitor that selectively targets the LMP7 subunit of immunoproteasomes being 20 to 40 fold more se lective for the LMP7 subunit than for the next most sensitive subunits, $\beta 5$ or LMP2 (Muchamuel et al., 2009). The molecular reason for the LMP7 selectivity of ONX 0914 has recently been elucidated by crys tallographic studies (Huber et al., 2012). The S1 pocket of LMP7 is more spacious compared to that of $\beta 5$. Thereby, the morpholine derivative adduct formation between the active site threonine and the pharma cophore of ONX 0914 would require a dislocation of Met45 in $\beta 5$ which would in turn result in energetically unfavorable major structural changes within the protein. Treatment with ONX 0914 was shown to attenuate several inflammatory diseases in mouse models at doses of less than one tenth of the maximum tolerated dose, a therapeutic window that is not achievable with nonselective inhibitors (Groettrup et al., 2010; Muchamuel et al., 2009; Basler et al., 2010; Basler et al., 2014; Basler et al., 2015; Mundt et al., 2016a). Additionally, under polarizing conditions in vitro, ONX 0914 suppressed the development of Th1 and Th17 cells and promoted Treg cell development without af fecting the differentiation of Th2 cells (Muchamuel et al., 2009; Kalim et al., 2012). Nota bene, these consequences of immunoproteasome 
a

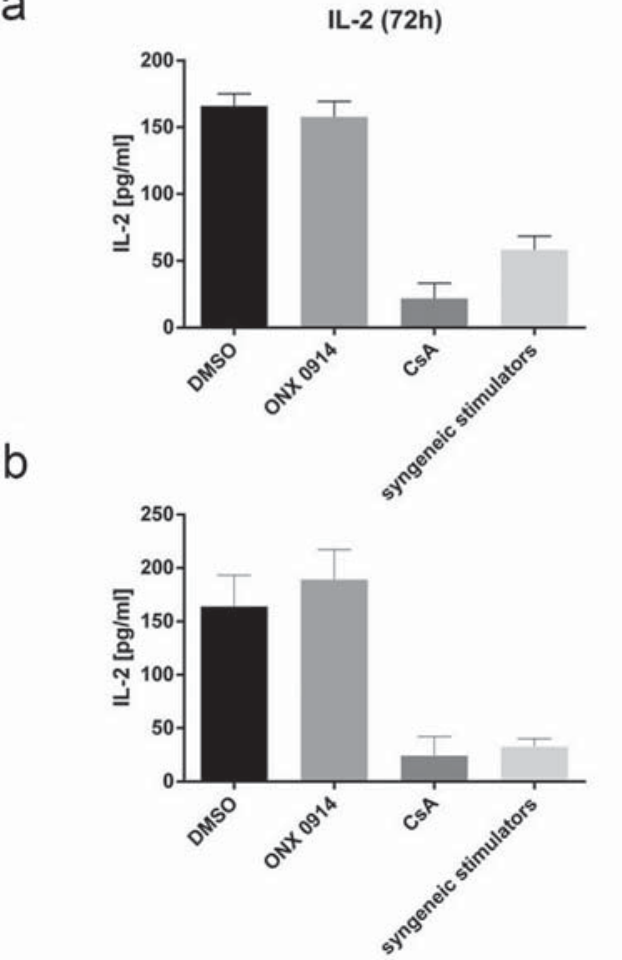

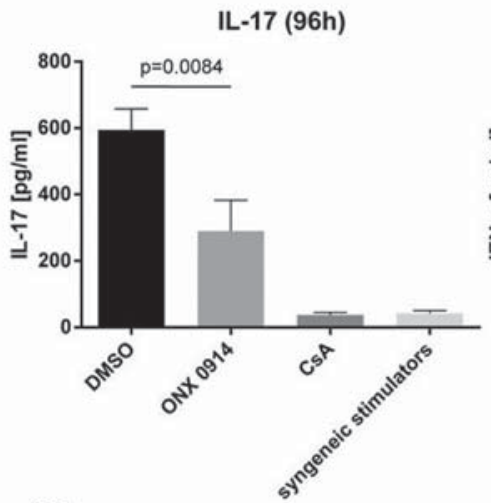
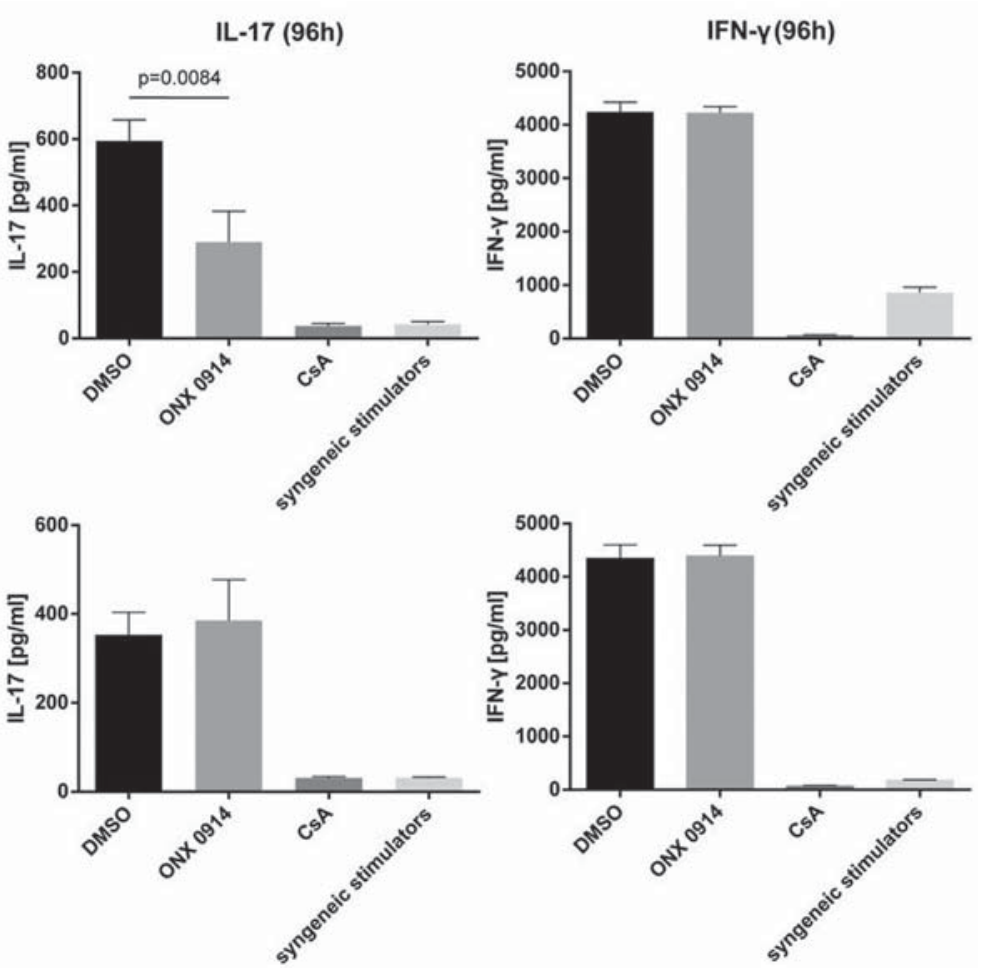

Fig. 2. Influence of ONX 0914 on allo-specific cytokine production in a mixed lymphocyte reaction. T cells magnetically isolated from bulk splenocytes of (a) C57BL/6 (H-2 ${ }^{\mathrm{b}}$ ) and (b) LMP7 / $\left(\mathrm{H}-2^{\mathrm{b}}\right)$ mice were treated with DMSO, ONX $0914(250 \mathrm{nM})$, or CsA $(1 \mu \mathrm{M})$ and cultured in the presence of allogeneic irradiated stimulator splenocytes from BALB/c (H-2 $\left.{ }^{\mathrm{d}}\right)$ mice for $7296 \mathrm{~h}$, as indicated. Syngeneic stimulator splenocytes (syngeneic stimulators) were used as negative control. Data show mean \pm SD of IL-2, IFN- $\gamma$, and IL-17 concentrations in the supernatant from triplicates and represent one out of two independent experiments as determined by ELISA. Data analyzed by student's $t$-test (two-tailed).

a

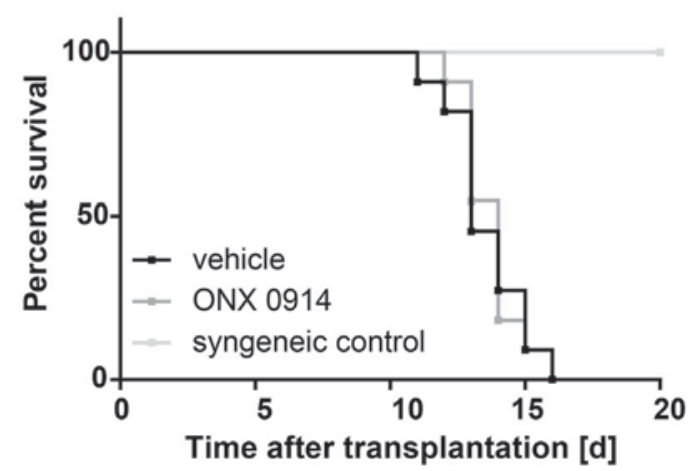

b

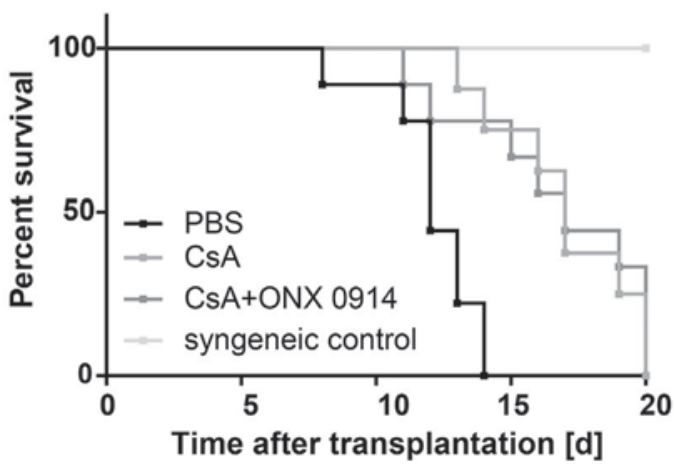

Fig. 3. Influence of LMP7 inhibition on MHC-mismatched skin allograft rejection. BALB/c recipient mice were transplanted with fully-thickness tail skin of C57BL/6 mice and (a) treated with vehicle (s.c.) or ONX 0914 (10 mg/kg, s.c.) every second day or (b) treated daily with PBS (i.p.) or CsA (5 mg/kg; i.p.) \pm ONX 0914 (10 mg/kg, s.c.) every second day from day 1 of transplantation onwards. Graft survival was monitored daily after removal of the bandage. Graphs show survival curves and represent pooled data from (a) three independent experiments $(\mathrm{n}=11$ per group and $\mathrm{n}=4$ syngeneic controls) or (b) two independent experiments $(\mathrm{n}=8-9$ per group and $\mathrm{n}=4$ syngeneic controls). With $\mathrm{p}(\mathrm{PBS} v \mathrm{~s}$. CsA) $=0.0007$ and $\mathrm{p}(\mathrm{PBS}$ vs. CsA + ONX 0914) $=0.0024$. Data were analyzed by Log-rank (Mantel-Cox) Test.

Table 1

Survival of C57BL/6 skin allografts in BALB/c recipients treated with ONX 0914.

\begin{tabular}{llll}
\hline Donor & Recipient & Treatment & Graft survival time (d) \\
\hline C57BL/6 & BALB/c & vehicle s.c. & $11,12,13,13,13,13,14,14,15,15,16$ \\
& & ONX 0914s.c. & $12,13,13,13,13,14,14,14,14,15,16$ \\
\hline
\end{tabular}

inhibition occurred in the absence of antigen presenting cells and were independent of the function of the immunoproteasome in antigen processing.

These findings suggest the immunoproteasome as a promising target for therapeutic intervention in several inflammatory disorders and encouraged us to investigate whether selective inhibition of LMP7 is able to prolong graft survival in two skin transplantation models. However, despite influencing allo specific Th17 cell responses in vitro, treatment of recipient mice with ONX 0914 had no influence on the allogeneic rejection response in vivo. 
Table 2

Survival of C57BL/6 skin allografts in BALB/c recipients treated with ONX 0914 in combination with low dose cyclosporine A.

\begin{tabular}{|c|c|c|c|c|}
\hline Donor & Recipient & Treatment & Graft survival time (d) & Median graft survival time $(\mathrm{d}) \pm \mathrm{SD}$ \\
\hline \multirow[t]{5}{*}{ C57BL/6 } & $\mathrm{BALB} / \mathrm{c}$ & PBS i.p. & $8,11,12,12,12,13,14,14$ & $12 \pm 1.8$ \\
\hline & & CsA i.p. & $13,14,16,17,17,19,20,20$ & $17 \pm 2.5$ \\
\hline & & CsA i.p. & $11,12,15,16,17,19,20,20,20$ & $17 \pm 3.2$ \\
\hline & & ONX & & \\
\hline & & 0914s.c. & & \\
\hline
\end{tabular}

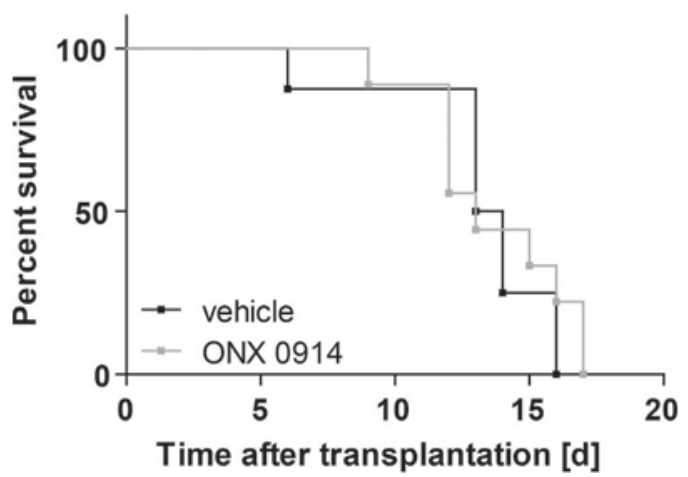

Fig. 4. Influence of LMP7 inhibition on multiple miHA mismatched skin allograft rejection. $\mathrm{C} 3 \mathrm{H}$ recipient mice were transplanted with fully-thickness tail skin of B10.Br donor mice and treated with vehicle (s.c.) or ONX $0914(10 \mathrm{mg} / \mathrm{kg}$, s.c.) every second day from day 1 of transplantation onwards. Graft survival was monitored daily after removal of the bandage. Graphs show survival curves and represent pooled data from two independent experiments ( $n=8-9$ per group). Data were analyzed by Log-rank (MantelCox) Test.

\section{Materials and methods}

\subsection{Mice}

C57BL/6 (H $\left.2^{\mathrm{b}}\right)$ mice, BALB/c (H $\left.2^{\mathrm{d}}\right)$ mice, C3H $\left(\mathrm{H} 2^{\mathrm{k}}\right)$, and B10.Br $\left(\mathrm{H} 2^{\mathrm{k}}\right)$ were originally purchased from Charles River, Germany. LMP7 gene targeted mice ( $\mathrm{H}^{\mathrm{b}}$ ) (Fehling, 1994) were provided by John Monaco (University of Cincinnati, Cincinnati, $\mathrm{OH}$ ). Mice were kept in a specific pathogen free facility and used at 810 weeks of age. Mouse experiments followed the Principles of Laboratory animal care and the German Law on the Protection of Animals. The animal experiments were approved by the Review Board of Regierungspräsidium Freiburg.

\subsection{Immunoproteasome inhibition}

For in vitro experiments, the LMP7 selective inhibitor ONX 0914 (formerly PR 957, contributed by Christopher J. Kirk, Kezar Life Science, South San Francisco, CA) was dissolved at a concentration of $10 \mathrm{mM}$ in DMSO and stored at $-80^{\circ} \mathrm{C}$. For in vivo proteasome inhibi tion, ONX 0914 was formulated in an aqueous solution of $10 \%(\mathrm{w} / \mathrm{v})$ sulfobutylether $\beta$ cyclodextrin (Captisol ${ }^{\circ}$ ) and $10 \mathrm{mM}$ sodium citrate (pH 6) referred to as vehicle and administered to mice as an s.c. bolus $(100 \mu \mathrm{l})$ dose of $10 \mathrm{mg} / \mathrm{kg}$.

\subsection{Cyclosporine A (CsA) treatment}

For in vitro experiments, CsA (Sigma) was dissolved at a con centration of $50 \mathrm{mM}$ in $\mathrm{EtOH}$ and stored at $-20^{\circ} \mathrm{C}$. For in vivo treatment, CsA (Sandimmune ${ }^{\circledR}$, Novartis) was formulated in PBS and administered to mice as an i.p. bolus $(100 \mu \mathrm{l})$ dose of $5 \mathrm{mg} / \mathrm{kg}$.

\subsection{Skin allograft transplantation}

Recipient BALB/c or C3H mice were anesthetized with a mixture of ketamine $(120 \mathrm{mg} / \mathrm{kg}$; i.p.) and xylazine $(10 \mathrm{mg} / \mathrm{kg}$, i.p.) in isotonic sterile saline solution and additionally received carprofen $(5 \mathrm{mg}$ / $\mathrm{kg}$, s.c.) to reduce pain on the day of transplantation and $24 \mathrm{~h}$ later. The dorsal skin was shaved and a $1 \mathrm{~cm}^{2}$ graft bed was prepared on the lateral back. Donor C57BL/6 or B10.Br mice were sacrificed and $1 \mathrm{~cm}^{2}$ full thickness tail skin grafts were prepared and transplanted to the beds on the back of the recipient mice. After removal of the bandage, graft survival was monitored daily and rejection was defined by complete destruction of the skin graft, as assessed by visual inspection.

\subsection{Mixed lymphocyte reaction}

Influence of ONX 0914 on allo specific T cell proliferation was as sessed by mixed lymphocyte reaction. MACS (Mouse Pan Isolation Kit II, Miltenyi) sorted splenic T cells originated from naïve C57BL/6 $(\mathrm{H}$ $\left.2^{\mathrm{b}}\right), \mathrm{BALB} / \mathrm{c}\left(\mathrm{H}^{\mathrm{d}}\right)$, or $\mathrm{LMP}^{-/-}\left(\mathrm{H} 2^{\mathrm{b}}\right)$ mice were used as responders, while residual splenocytes ( $\mathrm{T}$ cells) derived from C57BL/ 6 or BALB/c mice were used as stimulators. Responder $\mathrm{T}$ cells were carboxy fluorescein succinimidyl ester (CFSE) labeled as previously described (Moebius et al., 2010) and pulsed with DMSO or $250 \mathrm{nM}$ ONX 0914 in IMDM $10 \%$ FCS at $37^{\circ} \mathrm{C}$ for $2 \mathrm{~h}$ or continuously treated with $1 \mu \mathrm{M}$ CsA, respectively. $1 \times 10^{6}$ responder $\mathrm{T}$ cells were cultured with $1 \times 10^{5}$ irradiated (2000 rad) stimulators in IMDM $10 \%$ FCS in a 96 well plate. The cultures were incubated at $37{ }^{\circ} \mathrm{C}$ in $5 \% \mathrm{CO}_{2}$ for $7296 \mathrm{~h}$. Cell cul ture supernatants were analyzed for IL 2, IL 17, and IFN $\gamma$ by ELISA according to the manufacturer's protocol (eBioscience) and allo specific proliferation of $\mathrm{CD}^{+}{ }^{+}$and $\mathrm{CD} 8^{+} \mathrm{T}$ cells was determined by flow cy tometry.

\subsection{Flow cytometry}

Flow cytometry was performed as previously described (Mundt et al., 2015). Shortly, cells were stained with antibodies to CD4 (eBioscience, clone GK1.5), and CD8 (eBioscience, clone 53 6.7) in $50 \mu 1$ FACS buffer ( $2 \%$ FBS, $2 \mathrm{mM}$ EDTA, $2 \mathrm{mM} \mathrm{NaN}_{3}$ in PBS) at $4{ }^{\circ} \mathrm{C}$ for 20 min, washed two times and acquired with the Accuri ${ }^{\mathrm{TM}}$ C6 flow cytometer (BD Biosciences).

\subsection{Proteasome activity assay}

An MLR was setup as described above. $2 \mathrm{~h}$ and $24 \mathrm{~h}$ later, the cell permeable substrate MeoSuc GLF AMC (Harding et al., 1995) (Bachem)

Table 3

Survival of B10.Br allografts in C3H recipients treated with ONX 0914.

\begin{tabular}{llll}
\hline Donor & Recipient & Treatment & Graft survival time (d) \\
\hline B10.Br & C3H & vehicle s.c. & $6,13,13,13,14,14,16,16$ \\
& & ONX 0914s.c. & $9,12,12,12,13,13,15,16,17,17$ \\
\hline
\end{tabular}


(10 $\mathrm{mM}$ in DMSO) was added at $40 \mu \mathrm{M}$ (in PBS $+25 \mathrm{mM}$ HEPES) to the cells and incubated for $1 \mathrm{~h}$ at $37^{\circ} \mathrm{C}$. The fluorescence intensity in the wells containing the cells was measured at an excitation wavelength of $360 \mathrm{~nm}$ and emission wavelength of $465 \mathrm{~nm}$ (Infinite M200 pro, TEC AN). The fluorescence intensity in wells with stimulators alone was subtracted from values obtained with MLR.

\subsection{Statistical analysis}

The statistical significance was determined using the Student $t$ test, two way ANOVA or Log rank (Mantel Cox) test with two tailed P value. All statistical analyses were performed using GraphPad Prism Software (GraphPad, San Diego, CA). Statistical significance was achieved when $\mathrm{p}<0.05$. If not indicated otherwise, differences are not significant.

\section{Results}

\subsection{LMP7 inhibition has no influence on allo specific $T$ cell proliferation but reduces allo specific IL 17A production of $T$ cells in vitro}

In order to investigate the impact of the immunoproteasome in hibition on allogeneic immune responses, we analyzed the effect of ONX 0914 on T cell proliferation in a mixed lymphocyte reaction (MLR) in vitro. To this aim, we treated magnetically sorted splenic CFSE labeled responder T cells with ONX 0914 and cultured them in the presence of irradiated allogeneic stimulator splenocytes. We found that LMP7 inhibition had no influence on the percentage of proliferated $\mathrm{CD}^{+}{ }^{+}$or $\mathrm{CD}^{+}{ }^{+} \mathrm{T}$ cells after $96 \mathrm{~h}$ of allogenic stimulation (Fig. $1 \mathrm{a}$ and b) whereas cyclosporine A (CsA) effectively blocked allo specific pro liferation. Activity assays with a cell permeable fluorogenic substrate for the chymotrypsin like activity demonstrated that ONX 0914 was active in the MLR (Fig. 1c). Furthermore, we measured allo specific IL 2, IL 17, and IFN $\gamma$ release of T cells by ELISA. While ONX 0914 treatment did not reduce IL 2 or IFN $\gamma$ production it resulted in de creased IL 17A levels in the supernatant of the MLR (Fig. 2a). This effect was LMP7 specific since a reduction of IL 17A was not detected when T cells from $\mathrm{LMP7}^{-/-}$mice were treated with ONX 0914 (Fig. 2b).

\subsection{ONX 0914 treatment does not prolong graft survival in an MHC mismatched skin allograft transplantation model}

LMP7 inhibition was shown to suppress the differentiation of Th1 and Th17 cells in vitro and in vivo (Muchamuel et al., 2009; Basler et al., 2014; Kalim et al., 2012; Mundt et al., 2016b; Zilberberg et al., 2015). Consequently, the decreased allo specific Th17 differentiation of ONX 0914 treated cells in vitro prompted us to investigate the influence of LMP7 inhibition on allogeneic immune responses in vivo using a murine skin allograft transplantation model. For this purpose, C57BL/ 6 tail skin grafts were transplanted onto the back of $\mathrm{BALB} / \mathrm{c}$ mice. The recipient mice were treated with ONX 0914, at a dose previously shown to be effective in preventing autoimmunity, or vehicle (Captisol ${ }^{\circ}$ ), respec tively, and the graft was monitored daily for signs of rejection. Both vehicle and ONX 0914 treated mice rejected their allograft within 1116 days with a median graft survival time (MGST) of 13 and 14 days, respectively (Fig. 3a, Table 1), whereas syngeneic transplants were not rejected. Hence, no influence of LMP7 inhibition on the graft survival could be observed.

Next, we investigated whether ONX 0914 treatment in combination with a low dose CsA treatment could act synergistically in prolonging skin graft survival. CsA inhibits allo reactive Th1 responses, thus a combination of drugs could result in such a synergism. We compared skin allograft survival of BALB/c mice treated with $5 \mathrm{mg} / \mathrm{kg}$ CsA only or in combination with $10 \mathrm{mg} / \mathrm{kg}$ ONX 0914 (Fig. 3b, Table 2). We de tected a delayed rejection in mice treated with low dose CsA (MGST: 17 days) compared to the PBS treated control group (MGST: 12 days). However, additional ONX 0914 treatment did not further prolong allograft survival (MGST: 17 days). Hence, our data suggest that in hibiting the immunoproteasome is not effective in preventing acute $\mathrm{T}$ cell mediated allograft rejection in an MHC disparate skin transplan tation model.

\subsection{LMP7 inhibition does not prolong graft survival in a multiple miHA mismatch skin allograft transplantation model}

Primary skin grafts are known to be very sensitive to rejection compared to other organs and the effect of ONX 0914 might be too subtle to detect it in a transplantation model with a high degree of genetic mismatch as it is the case for the C57BL/6 to BALB/c strain combination (Fig. 3a). Hence, we decided to investigate the influence of LMP7 inhibition in a less sensitive multiple minor histocompatibility antigen (miHA) mismatch MHC matched transplantation model be tween $\mathrm{B} 10 . \mathrm{Br}\left(\mathrm{H}^{\mathrm{k}}\right)$ donor and $\mathrm{C} 3 \mathrm{H}\left(\mathrm{H}^{\mathrm{k}}\right)$ recipient mice. B10.Br tail skin grafts were transplanted onto the back of $\mathrm{C} 3 \mathrm{H}$ mice. The recipient mice were treated with $10 \mathrm{mg} / \mathrm{kg}$ ONX 0914 or vehicle, respectively, and the graft was monitored daily for signs of rejection. However, we could not detect any influence on graft survival in this model (Fig. 4, Table 3). Vehicle treated mice displayed a MGST of 13.5 days while ONX 0914 treatment resulted in a MGST of 13 days.

\section{Discussion}

Allograft rejection is a major threat to clinical organ transplantation and only due to immunosuppression over $90 \%$ of most organ trans plants survive (Winsett et al., 2001). Immunosuppressive drugs, how ever, affect not only the cells of the immune system, but have adverse side effects on other cells or tissues and long term administration of these compounds can cause nephrotoxicity, susceptibility to infection, and onset of diabetes (Schweer et al., 2014; Nankivell et al., 2004). This ultimately evokes the search for new and safe immunosuppressive drugs.

In the present study, we investigated the influence of ONX 0914, an LMP7 selective inhibitor, on skin allograft rejection. With the discovery of IL 17 producing $\mathrm{CD}^{+}{ }^{+} \mathrm{T}$ cells in 2005 a role of Th17 mediated al lograft rejection was suggested by a vast body of data (Itoh et al., 2011; Gorbacheva et al., 2010; Min et al., 2009). Th17/Treg ratios were found to be significantly higher during allograft rejection, suggesting that Th17 to Treg imbalance plays a role in the development of allograft rejection (Min et al., 2009). Thus, reversing this imbalance by LMP7 inhibition (Kalim et al., 2012) seemed to be a promising approach to interfere with allograft rejection. However, although we observed re duced IL 17A production in an MLR in vitro (Fig. 2), the influence of LMP7 inhibition was not sufficient to interfere with acute allogeneic responses during transplant rejection in vivo. Treatment of recipient mice with ONX 0914 did not prolong graft survival in a murine MHC mismatched skin allograft transplantation model (Fig. 3a) neither alone nor in combination with a low dose CsA treatment (Fig. 3b).

A reason for the insufficient suppression of allogeneic immune re sponses by ONX 0914 treatment might be explained by the fact, that the fully MHC disparate skin allograft transplantation is very sensitive to rejection eliciting strong allogeneic immune responses. Moreover, it has been shown that $\mathrm{T}$ cell derived IL 17 is critical for spontaneous rejec tion of miHA but not MHC disparate skin grafts (Vokaer et al., 2010). These facts suggest that the effect of ONX 0914 on allogeneic immune responses might not be strong enough to prolong graft survival in this model. Therefore, we investigated the influence of immunoproteasome inhibition in a less sensitive skin transplantation model between mul tiple miHA disparate mice (C3H and B10.Br). However, ONX 0914 treatment also failed to protract graft rejection in this model (Fig. 4).

Hence, immunoproteasome inhibition does not appear as a suitable approach to treat acute $\mathrm{T}$ cell mediated skin allograft rejection. Whether this approach could be effective in other models of graft re jection would have to be a subject of future investigations. 
Interestingly, Zilberberg et al. reported LMP7 inhibition to be ef fective in ameliorating graft versus host disease (GvHD) in a miHA disparate murine blood and marrow transplant (BMT) model by de creasing endogenous miHA presentation and consequently reducing allogeneic stimulation and cytokine production (Zilberberg et al., 2015). In contrast to our study, Zilberberg et al. used a daily or even $2 \mathrm{x}$ daily treatment regimen, which might have a stronger effect than our treatment strategy. We have chosen to treat the mice three times a week with $10 \mathrm{mg} / \mathrm{kg}$, a dose, which was proven to be effective in auto immune models like for example EAE. This dose is still LMP7 selective and well below the maximum tolerated dose (MTD) of $30 \mathrm{mg} / \mathrm{kg}$ in mice (Muchamuel et al., 2009; Basler et al., 2010; Basler et al., 2014). However, the in vivo effect of ONX 0914 on GVHD reported in the study of Zilberberg et al. is rather moderate with all mice still succumbing to GVHD but with slightly delayed kinetics.

More recently, E. Sula Karreci et al. could show that a newly de scribed LMP7 specific immunoproteasome inhibitor (designated DPLG3) prolonged survival of cardiac allografts in mice and allowed long term survival when combined with single dose CTLA4 Ig treat ment (Sula Karreci et al., 2016). Importantly, DPLG3 is a non covalent N,C capped dipeptide inhibitor and hence has a different mode of ac tion compared to ONX 0914. Although DPLG3 is reported to be a very potent inhibitor with a low $\mathrm{IC}_{50}$ and a high selectivity index, the au thors used a rather high dose for their in vivo experiments. On the other hand, they failed to show that this treatment regimen is still LMP7 selective or to determine the MTD for this compound. Moreover, even with a dose of $25 \mathrm{mg} / \mathrm{kg}$ the inhibitor by itself did not prolong cardiac allograft survival. Only in combination with CTLA4 Ig, which itself significantly prolonged the survival time by about 50 days, DPLG3 was able to inhibit allograft rejection.

Collectively, LMP7 inhibition seems to have a minor to moderate potential in reducing acute allogeneic $\mathrm{T}$ cell responses at least when given in combination with other drugs of current maintenance regimens like e.g. calcineurin inhibitors, mTOR inhibitors, anti proliferative agents (e.g. mycophenolic acid), costimulation blockers, and corticos teroids.

Taken together, immunoproteasome inhibition does not appear to be a suitable approach to treat acute $\mathrm{T}$ cell mediated skin allograft re jection.

\section{Conflict of interest statement}

The authors declare no commercial or financial conflict of interest.

\section{Authorship}

S.M. designed and performed experiments and wrote the manu script. B.S. provided advice and help for skin allograft transplantation and corrected and refined the manuscript. M.B. performed experiments, supervised the project and corrected and refined the manuscript. M.G. supervised the project and corrected and refined the manuscript.

\section{Funding sources}

This work was funded by the German Research Foundation grant BA 4199/2 1 to MB and GR 1517/14 1 to MG, SwissLife Jubiläumsstiftung to MB, and grant Nr. 2017 A28 by the Else Kröner Fresenius Stiftung to MG.

\section{Acknowledgements}

Christine Appelt is acknowledged for skin transplantation instruc tions and John Monaco for the contribution of LMP7 ${ }^{-/-}$mice. We thank Christopher J. Kirk (Kezar Life Science) for the generous con tribution of ONX 0914.

\section{References}

Basler, M., et al., 2010. Prevention of experimental colitis by a selective inhibitor of the immunoproteasome. J. Immunol. 185 (1), 634-641.

Basler, M., Kirk, C.J., Groettrup, M., 2013. The immunoproteasome in antigen processing and other immunological functions. Curr. Opin. Immunol. 25 (1), 74-80.

Basler, M., et al., 2014. Inhibition of the immunoproteasome ameliorates experimental autoimmune encephalomyelitis. EMBO Mol. Med. 6 (2), 226-238.

Basler, M., et al., 2015. The immunoproteasome: a novel drug target for autoimmune diseases. Clin. Exp. Rheumatol. 33, 74-79 4 Suppl. 92.

Fehling, H.J., 1994. MHC class I expression in mice lacking proteasome subunit LMP7 Science $265,1234-1237$.

Goldberg, A.L., et al., 2002. The importance of the proteasome and subsequent proteolytic steps in the generation of antigenic peptides. Mol. Immunol. 39 (3-4), 147-164.

Gorbacheva, V., et al., 2010. Interleukin-17 promotes early allograft inflammation. Am. J. Pathol. 177 (3), 1265-1273.

Groettrup, M., et al., 2001. Interferon-gamma inducible exchanges of $20 \mathrm{~S}$ proteasome active site subunits: why? Biochimie 83 (3-4), 367-372.

Groettrup, M., Kirk, C.J., Basler, M., 2010. Proteasomes in immune cells: more than peptide producers? Nat. Rev. Immunol. 10 (1), 73-78.

Harding, C.V., et al., 1995. Novel dipeptide aldehydes are proteasome inhibitors and block the MHC-I antigen-processing pathway. J. Immunol. 155 (4), 1767-1775.

Huber, E.M., et al., 2012. Immuno- and constitutive proteasome crystal structures reveal differences in substrate and inhibitor specificity. Cell 148 (4), 727-738.

Itoh, S., et al., 2011. Interleukin-17 accelerates allograft rejection by suppressing regulatory T cell expansion. Circulation 124 (11 Suppl), S187-96.

Jiang, S., Herrera, O., Lechler, R.I., 2004. New spectrum of allorecognition pathways: implications for graft rejection and transplantation tolerance. Curr. Opin. Immunol. 16 (5), 550-557.

Kalim, K.W., et al., 2012. Immunoproteasome subunit LMP7 deficiency and inhibition suppresses Th1 and Th17 but enhances regulatory T cell differentiation. J. Immunol. 189 (8), 4182-4193.

Li, T., et al., 2010. Phase II study of the proteasome inhibitor bortezomib (PS-341, Velcade) in chemotherapy-naive patients with advanced stage non-small cell lung cancer (NSCLC). Lung Cancer 68 (1), 89-93.

Min, S.I., et al., 2009. Sequential evolution of IL-17 responses in the early period of allograft rejection. Exp. Mol. Med. 41 (10), 707-716.

Miura, M., El-Sawy, T., Fairchild, R.L., 2003. Neutrophils mediate parenchymal tissue necrosis and accelerate the rejection of complete major histocompatibility complexdisparate cardiac allografts in the absence of interferon-gamma. Am. J. Pathol. 162 (2), 509-519.

Moebius, J., et al., 2010. Immunoproteasomes are essential for survival and expansion of T cells in virus-infected mice. Eur. J. Immunol. 40 (12), 3439-3449.

Muchamuel, T., et al., 2009. A selective inhibitor of the immunoproteasome subunit LMP7 blocks cytokine production and attenuates progression of experimental arthritis. Nat. Med. 15 (7), 781-787.

Mundt, S., Groettrup, M., Basler, M., 2015. Analgesia in mice with experimental meningitis reduces pain without altering immune parameters. ALTEX 32 (3), 183-189.

Mundt, S., et al., 2016a. Inhibition and deficiency of the immunoproteasome subunit LMP7 attenuates LCMV-induced meningitis. Eur. J. Immunol. 46 (1), 104-113.

Mundt, S., et al., 2016b. Inhibiting the immunoproteasome exacerbates the pathogenesis of systemic Candida albicans infection in mice. Sci. Rep. 6, 19434.

Nankivell, B.J., et al., 2004. Calcineurin inhibitor nephrotoxicity: longitudinal assessment by protocol histology. Transplantation 78 (4), 557-565.

Raghavan, R., et al., 2010. Bortezomib in kidney transplantation. J. Transplant 2010.

Richardson, P.G., et al., 2005. Bortezomib or high-dose dexamethasone for relapsed multiple myeloma. N. Engl. J. Med. 352 (24), 2487-2498.

Richardson, P.G., et al., 2006. Frequency: characteristics, and reversibility of peripheral neuropathy during treatment of advanced multiple myeloma with bortezomib. J. Clin. Oncol. 24 (19), 3113-3120.

Schweer, T., et al., 2014. High impact of rejection therapy on the incidence of posttransplant diabetes mellitus after kidney transplantation. Clin. Transplant 28 (4), 512-519.

Sula Karreci, E., et al., 2016. Brief treatment with a highly selective immunoproteasome inhibitor promotes long-term cardiac allograft acceptance in mice. Proc. Natl. Acad. Sci. U. S. A. 113 (52), E8425-E8432.

Vogelbacher, R., et al., 2010. Bortezomib and sirolimus inhibit the chronic active antibody-mediated rejection in experimental renal transplantation in the rat. Nephrol. Dial. Transplant. 25 (11), 3764-3773.

Vokaer, B., et al., 2010. Critical role of regulatory T cells in Th17-mediated minor antigen-disparate rejection. J. Immunol. 185 (6), 3417-3425.

Walsh, R.C., et al., 2010. Proteasome inhibitor-based primary therapy for antibodymediated renal allograft rejection. Transplantation 89 (3), 277-284.

Winsett, R.P., et al., 2001. Immunosuppressant side effect profile does not differ between organ transplant types. Clin. Transplant 15 (Suppl. 6), 46-50 (p).

Wood, K.J., Bushell, A., Hester, J., 2012. Regulatory immune cells in transplantation. Nat. Rev. Immunol. 12 (6), 417-430.

Zilberberg, J., et al., 2015. Inhibition of the Immunoproteasome Subunit LMP7 with ONX 0914 Ameliorates Graft-versus-Host Disease in an MHC-Matched Minor

Histocompatibility Antigen-Disparate Murine Model. Biol. Blood Marrow Transplant. 21 (9), 1555-1564. 